\title{
Correction to: Facile synthesis of carbon-coated layered double hydroxide and its comparative characterisation with Zn-AI LDH: application on crystal violet and malachite green dye adsorption-isotherm, kinetics and Box-Behnken design
}

Giphin George ${ }^{1}$ Manickam Puratchiveeran Saravanakumar ${ }^{1}$

Published online: 7 September 2018

(C) Springer-Verlag GmbH Germany, part of Springer Nature 2018

Correction to: Environmental Science and Pollution Research

https://doi.org/10.1007/s11356-018-3001-3

The original publication of this paper contains an error.

The correct Figure 8 is shown in this paper.

The original article has been corrected.

The online version of the original article can be found at https://doi.org/ 10.1007/s11356-018-3001-3

Manickam Puratchiveeran Saravanakumar saravanakumar05@gmail.com

Giphin George

giphingeorge@gmail.com

1 School of Civil and Chemical Engineering, Vellore Institute of Technology, Vellore Campus, Vellore, India 
(a) Yoshida H-bonding interaction

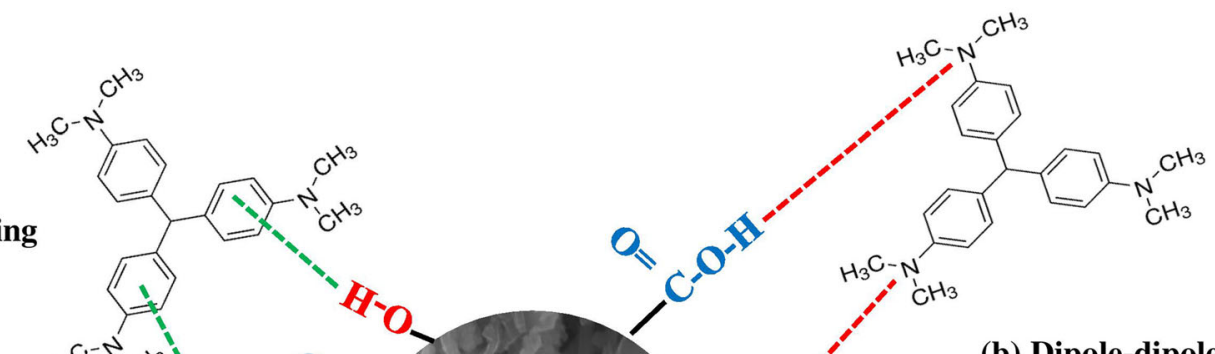

(b) Dipole-dipole H-bonding interaction

(c) Electrostatic interaction

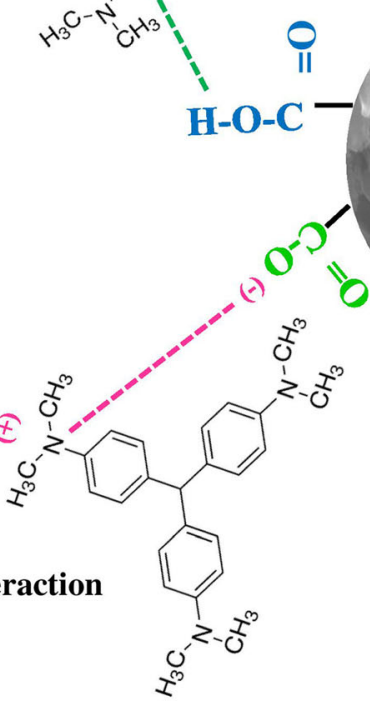

(e) Pore filling

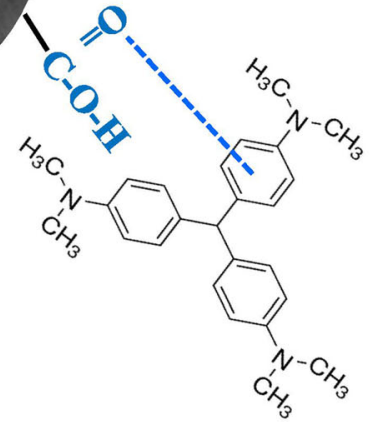

(d) $n-\pi$ interaction

(a)

(a) Yoshida H-bonding interaction

(c) Electrostatic interaction

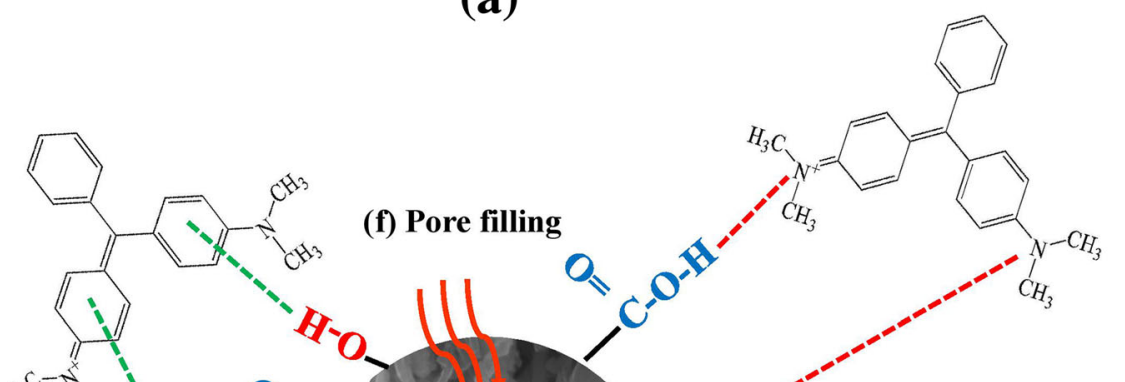

(b) Dipole-dipole H-bonding interaction

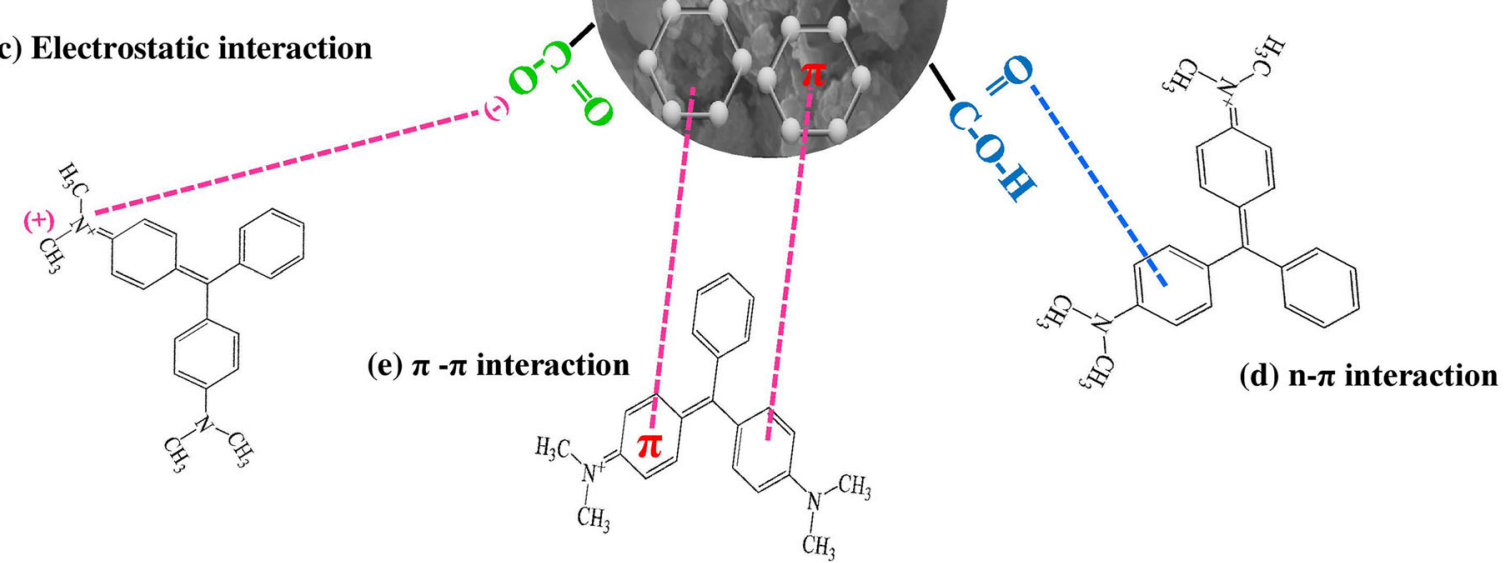

(b)

Fig. 8 Proposed adsorption mechanisms of (a) CV and (b) MG onto the C-Zn-Al LDH 